\title{
Retour d'expérience sur Covidom : une solution de télésurveillance pour les patients porteurs ou suspectés Covid-19
}

\section{Feedback on COVIDOM: a Telemedicine Solution for Home Monitoring of Patients with Mild COVID-19}

\author{
Collaboration AP-HP/universités/Inserm Covid-19 \\ Reçu le 15 juillet 2020 ; accepté le 4 août 2020 \\ (c) SFMU et Lavoisier SAS 2020
}

Collaboration AP-HP/universités/Inserm Covid-19

\section{Comité d'écriture}

Yordanov Youri ${ }^{1,2}$, Dechartres Agnès ${ }^{2,3}$, Lescure François-Xavier ${ }^{4,5}$, Apra Caroline ${ }^{6}$, Villie Pascaline ${ }^{7}$, Marchand-Arvier Jérôme ${ }^{8}$, Dinh Aurélien $^{8}$, Jourdain Patrick ${ }^{9,10}$, Debuc Erwan ${ }^{1}$

1 Service d'accueil des urgences, hôpital Saint-Antoine, Assistance publique-Hôpitaux de Paris (APHP), Sorbonne Université, Paris, France

${ }^{2}$ Inserm, UMR-S 1136, institut Pierre-Louis d'épidémiologie et de santé publique, Sorbonne Université, Paris, France

${ }^{3}$ Département de santé publique, centre de pharmacoépidémiologie de l'AP-HP (Cephepi), hôpital Pitié-Salpêtrière, AP-HP, Sorbonne Université, Paris, France

4 Service de maladies infectieuses et tropicales, AP-HP, BichatClaude-Bernard University Hospital, Paris, France

${ }^{5}$ Infections Antimicrobials Modelling Evolution (IAME), UMR 1137, University of Paris, Paris, France

6 Service de neurochirurgie, hôpital Pitié-Salpêtrière, AP-HP, Sorbonne Université, Paris, France

${ }_{8}^{7}$ Assistance publique-Hôpitaux de Paris, Paris, France

8 Service de maladies infectieuses, Hôpital Raymond-Poincaré, Garches, AP-HP, Paris Saclay University, Paris, France

${ }^{9}$ DMU COREVE, GHU Paris Saclay, AP-HP, Paris, France

${ }^{10}$ Inserm, UMR-S 999, IHU TORINO (thorax innovation), France

\section{Comité data-sciences}

Caroline Apra, Charlotte Caucheteux, Alexandre Gramfort, Luc Jaulmes, Jenny Mansour, Arthur Mensch

Comité scientifique

Amélie Aime-Eusebi, Caroline Apra, Alexandre Bleibtreu, Erwan Debuc, Agnès Dechartres, Laurène Deconinck, Aurélien Dinh, Patrick Jourdain, Christine Katlama, Josselin Lebel, François-Xavier Lescure, Youri Yordanov

\section{Comité directeur du centre régional Covidom}

Yves Artigou, Amélie Banzet, Élodie Boucheron, Christiane Boudier, Édouard Buzenac, Marie-Claire Chapron, Dalhia Chekaoui, Laurent De Bastard, Erwan Debuc, Aurélien Dinh, Alexandre Grenier, Pierre-Étienne Haas, Julien Hody, Michèle Jarraya, Patrick Jourdain, Louis Lacaille, Aurélie Le Guern, Jeremy Leclert, Fanny Male, Jérôme MarchandArvier, Emmanuel Martin-Blondet, Apolinne Nassour, Oussama Ourahou, Thomas Penn, Ambre Ribardiere, Nicolas Robin, Camille Rouge, Nicolas Schmidt, Pascaline Villie

Centre de pharmacoépidémiologie de l'AP-HP (Cephépi) Sofia Zemouri

ITS, médecins, étudiants en santé et bénévole Covidom Liste en annexe 1, disponible à https://dispose.aphp.fr/u/ nC31Y16t9XWOLfVz/04\%20Annexe\%201.doc?1
Résumé Covidom est une plateforme de télésurveillance destinée aux patients suspects ou atteints de Covid-19, ne nécessitant pas une hospitalisation au décours de leur prise en charge initiale. Cet outil a été coconstruit avec tous les acteurs du soin (ville, hôpital, institutions). Il se compose d'une application Web et d'un centre de télésurveillance permettant de surveiller à distance les patients atteints de Covid-19. Cette surveillance est faite à l'aide de brefs questionnaires quotidiens, autoadministrés et standardisés. Actuellement, plus de 60000 patients ont achevé leur suivi dans Covidom. Covidom est une solution innovante permettant la surveillance à domicile des patients atteints d'une forme légère de Covid- 19.

Mots clés Covid-19 · Infection à coronavirus · Surveillance à distance $\cdot$ Télémédecine

Abstract COVIDOM is a telemonitoring platform that has been designed for patients suspected or affected by COVID19 and who do not require hospitalization. This tool has been co-constructed with all healthcare stakeholders (regional agencies, hospitals, and family physicians). It brings together a web application and a remote monitoring center for home monitoring of patients suffering from Covid-19. Monitoring is done via brief and standardized daily questionnaires. Currently, more than 60,000 patients have completed their follow-up in COVIDOM. COVIDOM is an innovative solution for home monitoring of patients with mild forms of COVID-19.

Keywords COVID-19 · Coronavirus disease · Home monitoring $\cdot$ Telemedicine

\section{Introduction}

En l'espace de quelques mois, la maladie à coronavirus (Covid-19) est passée d'un foyer isolé d'infections respiratoires dues à un virus émergent dans le Hubei (Chine) à une pandémie mondiale ayant entraîné le confinement de près de 
la moitié de l'humanité [1-3]. Les caractéristiques cliniques des patients atteints de la Covid-19 ont été largement décrites. Une immense majorité de patients présentent des formes légères ou asymptomatiques de la maladie, les complications et les décès survenant essentiellement chez des patients âgés ou présentant des comorbidités telles que l'obésité, le diabète ou encore l'insuffisance rénale chronique [4-12]. Hormis le défi thérapeutique que constitue toute maladie infectieuse émergente, la transmission exponentielle de celle-ci à l'ensemble d'une population non protégée a menacé la stabilité de nos systèmes de santé. Ces derniers étant régulièrement décrits comme au bord de la rupture, leur capacité à gérer cette nouvelle crise aux proportions hors normes était remise en question et poussait à imaginer des solutions innovantes pour préserver les structures hospitalières [13-17].

Afin de permettre aux hôpitaux de se concentrer sur les patients fragiles et les plus gravement malades, ceux ne présentant aucun signe de gravité étaient pris en charge de manière ambulatoire [8]. Cependant, le risque d'aggravation rapide (estimé à 10-15\% des cas) et les nombreuses inconnues persistantes autour de cette infection nouvelle rendaient une surveillance nécessaire [8-10,18,19]. Diverses solutions ont été envisagées, mais toutes reposaient sur la prise en charge individuelle de chaque patient par un médecin (généraliste ou infectiologue) [20,21]. Or en situation de pandémie, comme dans toute situation sanitaire exceptionnelle, il convient d'optimiser les ressources [20,22-24]. Ainsi, pour offrir des alternatives aux patients tout en préservant les ressources médicales aux situations qui le nécessitent, l'Assistance publique-Hôpitaux de Paris (APHP), en collaboration avec les organisations régionales de médecins généralistes libéraux (Union régionale des professionnels de santé ; URPS Médecins) et Nouveal e-santé, a rapidement développé et déployé une solution de télésurveillance à distance nommée Covidom. Cet outil, via une application Web, permet de surveiller à distance les patients atteints de Covid-19 présentant des symptômes bénins à l'aide de questionnaires de surveillance quotidiens, autoadministrés, brefs et standardisés.

Notre objectif est ici de présenter la solution Covidom, l'organisation de la plateforme de télésurveillance ainsi que l'application et les principales caractéristiques des patients ayant bénéficié de cette prise en charge.

\section{Description de Covidom}

Covidom est la combinaison d'une application, facile d'utilisation pour les patients, et d'un centre de télésurveillance unique pour la région Île-de-France (Fig. 1). Il s'agit d'une solution qui a été rapidement imaginée, construite et déployée de manière conjointe par l'ensemble des acteurs du soin francilien, à savoir les médecins de ville (URPS Médecins), l'AP-HP et l'ARS Île-de-France. Entre l'analyse des besoins et le déploiement de la première version, il s'est passé une semaine (du 2 mars au 9 mars 2020). Puis au bout d'une deuxième semaine, l'ensemble des structures publiques et privées en faisant la demande avaient la possibilité d'inclure des patients.

Les patients inscrits dans Covidom, s'ils remplissent les critères d'éligibilité suivants :

- tableau clinique évocateur de Covid-19 (suspect ou confirmé), après évaluation médicale (définition des cas selon Santé publique France : toute personne présentant des signes cliniques d'infection respiratoire aiguë [dyspnée et/ou toux] avec de la fièvre [ou une sensation de fièvre], sans autre cause identifiée [par exemple, pneumonie bactérienne, insuffisance cardiaque aiguë, etc.]) [25] ;

- avec des symptômes bénins, et sans indication d'hospitalisation (selon le jugement du praticien évaluateur) ;

- possédant un Smartphone, une tablette ou un ordinateur et étant capable de l'utiliser.

Ils pouvaient être inscrits dans le cadre de leur prise en charge initiale (en ambulatoire après un passage aux urgences, un appel au 15 ou une consultation avec un médecin généraliste) ou à la sortie d'une hospitalisation due à la Covid-19. L'inscription se faisait en deux étapes. D'abord, les patients éligibles étaient enregistrés par le médecin, après une présentation de la solution Covidom et après avoir donné leur consentement oral à participer. Le médecin saisissait les principales caractéristiques du patient (âge, sexe, date des premiers symptômes) et niveau de risque. Les patients étaient considérés à haut risque s'ils avaient des antécédents de maladie cardiovasculaire, du diabète, une insuffisance respiratoire chronique, une immunodépression (transplantation, cancer en cours de traitement, infection par le VIH non contrôlée...), une grossesse au troisième trimestre ou s'ils étaient âgés de plus de 65 ans. Puis les patients recevaient un lien d'inscription par un message sur leur mobile ou un courriel. À l'aide du lien, ils devaient confirmer (ou non) l'inscription, la compléter et donner leur consentement électronique à participer au programme de télésurveillance Covidom. Les patients étaient informés du stockage de leurs données et de leur utilisation potentielle, de manière anonymisée, à des fins de recherche. Cette utilisation a été approuvée par le comité scientifique et éthique de l'AP-HP (IRB00011591).

L'application a été conçue pour être intuitive et simple d'utilisation pour tous les utilisateurs (patients et médecins inclueurs). Selon leur profil de risque, les patients reçoivent un ou deux questionnaires quotidiens. Ils sont composés de moins de dix questions courtes et standardisées. Les questionnaires sont disponibles en ligne ou via l'application. Les patients sont informés par message mobile ou par e-mail de la mise à disposition de tout nouveau questionnaire. En cas de non-réponse au questionnaire dans l'heure qui suit le 
Figure 1: Description de COVIDOM

COMMENT S'ORGANISE LA PRISE EN CHARGE DES PATIENTS?

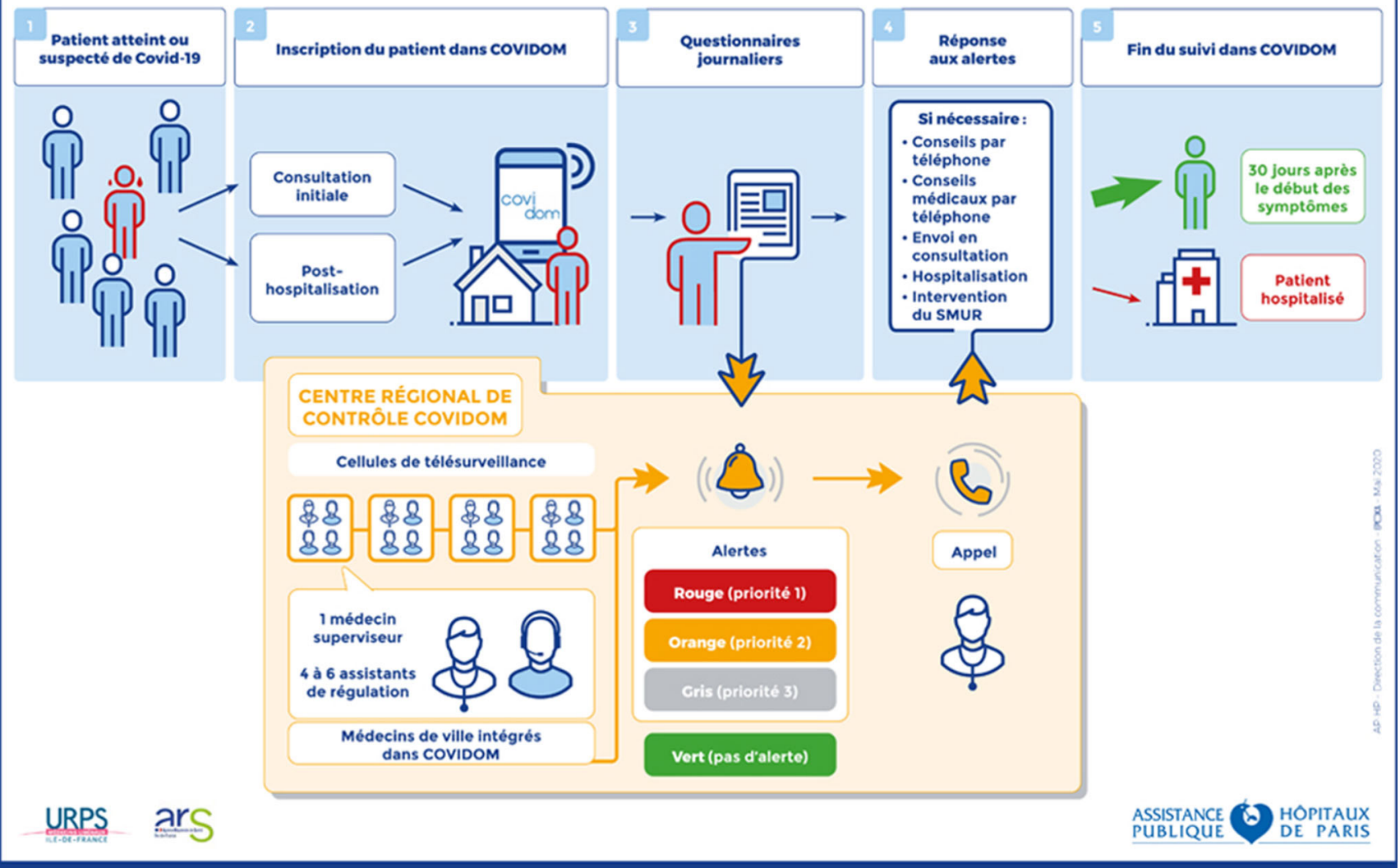

Fig. 1 Description de Covidom

message initial, un rappel est alors envoyé. Les patients renseignaient leur fréquence respiratoire et la fréquence cardiaque, la température, une éventuelle dyspnée, des nausées, un éventuel malaise et des difficultés éventuelles à gérer le confinement. Les réponses à ces questions pouvaient déclencher différents types d'alertes au centre de télésurveillance. Ces questionnaires ont été élaborés et testés par un panel pluridisciplinaire de professionnels de santé (médecins urgentistes, infectiologues, médecins généralistes, spécialistes de la télésurveillance). Les patients étaient également invités à répondre à trois autres questionnaires médicaux au cours de leur suivi : un, le jour de l'enregistrement pour recueillir les principales informations médicales et les facteurs de risque, un autre à deux semaines des premiers symptômes et un dernier questionnaire à la fin du suivi (en général à j30).

Dans l'application, les patients pouvaient également trouver plusieurs documents contenant des informations sur le virus, sur la manière de limiter la transmission, sur les méthodes de mesure de la température/fréquence cardiaque/fréquence respiratoire et enfin un document sur la manière de demander un soutien psychologique s'ils en exprimaient le besoin.

En cas d'urgence immédiate, il était conseillé aux patients de contacter directement le service d'aide médicale urgente (Samu) en composant le 15.

Un centre de télésurveillance régional, établi dans l'un des sites de l'AP-HP, était ouvert de 8 à 20 h, 7 jours sur 7 , afin de prendre en charge l'ensemble des patients utilisant Covidom dans la région parisienne (12 millions d'habitants). Il s'articulait autour du concept de cellules de télésurveillance autonomes. Chaque cellule était composée de quatre à six intervenants formés à la télésurveillance (ITS) et d'un médecin superviseur. Les cellules avaient pour mission de traiter les alertes générées par les réponses des patients. Celles-ci étaient classées en quatre catégories, à l'aide d'un algorithme automatisé :

- pas d'alerte (vert) : tout est considéré comme normal, aucune action n'est nécessaire ;

- alerte orange : certaines réponses dépassent un seuil prédéfini. Ces alertes nécessitent une réponse du centre de télésurveillance ; 
- alertes rouges : certaines des réponses suggèrent que la santé du patient pourrait se détériorer. Ces alertes nécessitent une réponse du centre de télésurveillance, ces alertes sont à prioriser ;

- alertes grises : les patients n'ont pas répondu au questionnaire. Ces alertes nécessitent une réponse du centre de contrôle, et les patients doivent être appelés, mais le niveau de priorité est faible.

Pour traiter les alertes, les ITS accédaient au dossier du patient et les contactaient par téléphone. Si nécessaire, le médecin superviseur de la cellule pouvait intervenir et directement évaluer le patient par téléphone. Une alerte était considérée comme traitée dès que l'ITS ou le médecin proposait une solution au patient : conseil général, conseil médical, orientation vers son médecin traitant, hospitalisation ou mise en contact avec le Samu. Le Samu, les services d'urgence ou les services de maladies infectieuses pouvaient être contactés directement par le centre à l'aide de numéros de téléphone dédiés. Enfin, si les patients en exprimaient le besoin, ils étaient orientés vers une cellule de soutien psychologique spécifiquement créée sur place.

Les médecins des cellules de contrôle et les ITS étaient des volontaires d'horizons différents. Tous recevaient une formation théorique et pratique. Celle-ci dépendait de leur profil. Ils ne recevaient aucune incitation financière, mais des repas ou des solutions de transport ont été proposés. Tous les volontaires ont signé un contrat individuel avec l'AP-HP pour des raisons de confidentialité, d'assurance et de responsabilité.

\section{Caractéristiques des patients inclus}

Pour cette description, nous avons inclus tous les patients inscrits sur la plateforme Covidom, du 9 mars au 7 mai 2020, qui avaient plus de 18 ans le jour de l'inscription, qui ont été inclus au cours de leur prise en charge initiale et qui ont rempli le questionnaire médical.

Nous avons collecté les données suivantes :

- âge et sexe ;

- poids et taille (permettant le calcul de l'indice de masse corporelle [IMC]) ;

- principales pathologies chroniques : diabète, hypertension, insuffisance rénale chronique, insuffisance cardiaque, pathologie coronarienne, cancer en cours de traitement, bronchopneumopathie obstructive, asthme ;

- consommation de tabac ;

- date des premiers symptômes et types des symptômes (initiaux, à j14 et à j30) ;

- confirmation du diagnostic : infection confirmée par un test (reverse transcriptase, réaction en chaîne par polymérase [RT-PCR]) ou pas. Ces informations ont été recou- pées avec l'entrepôt de données de l'AP-HP. Nous avons également recueilli si les patients ont passé une radiographie pulmonaire ou un scanner thoracique.

$\mathrm{Au}$ cours des trois premiers mois d'utilisation de la solution, 55516 patients atteints d'une forme légère de Covid-19 ont été enregistrés dans Covidom. Ces patients ont été recrutés par 1709 médecins hospitaliers et par 2131 généralistes. Une très grande majorité des patients ont été recrutés dans le cadre de leur prise en charge ambulatoire (87,5\%, 48 590/55 516). Au total, 27549 d'entre eux étaient des adultes et avaient rempli le questionnaire médical. Près d'un patient sur cinq $(17,6 \% ; n=4916 / 27549)$ a été inclus par un Samu. La plateforme a permis de traiter plus de 270000 alertes en moins de cinq minutes en moyenne. Parmi les alertes déclenchées, 352 ont mené à un contact avec le Samu départemental.

Parmi les 27549 patients, 63,1\% étaient des femmes, et l'âge moyen était de $43 \pm 14$ ans. La majorité des patients avaient moins de 60 ans $(n=24156 ; 88,0 \%)$. L'IMC médian était de $24,9 \mathrm{~kg} / \mathrm{m}^{2}$ (interquartile : $22,1-28,5$ ), avec près de $30 \%(n=8237)$ des patients en surpoids $(25<$ IMC $<30)$ et $18,1 \%(n=4987)$ obèses avec un IMC supérieur ou égal à 30 (Tableau 1). Les principaux antécédents rapportés par les patients étaient l'asthme, l'hypertension et le diabète dans, respectivement, $12,8 \%(n=3511), 12,6 \%(n=3457)$ et $4,9 \%(n=1349)$ des cas.

Le délai médian entre l'apparition des symptômes de Covid-19 et l'enregistrement dans Covidom était de quatre jours (interquartile [IQR] : 2-7 jours). Dans la majorité des cas, les patients ont signalé de la fatigue $(86,4 \% ; n=23$ $639)$ et de la toux $(63,9 \% ; n=17591)$. Les autres symptômes les plus fréquents étaient les frissons $(55,6 \% ; n=$ $15311)$ et/ou la fièvre $(49,2 \% ; n=13559)$, des myalgies $(54,9 \% ; n=15126)$ ainsi qu'une dyspnée $(48,8 \% ; n=$ 13438 ). Il est intéressant de noter qu'un tiers des patients ont déclaré une anosmie $(31,8 \% ; n=8770)$ ou une agueusie $(32,0 \% ; n=8822)$, et $10301(37,4 \%)$ au moins l'une des deux. Un quart $(24,7 \% ; n=6808)$ des patients déclarent avoir été testés (test de référence, soit RT-PCR sur écouvillonnage nasopharyngé), tandis que $10,8 \%(n=2690)$ et $4,9 \%(n=1219)$ des patients ont passé une TDM thoracique et une radiographie pulmonaire.

\section{Discussion}

À notre connaissance, Covidom est la solution de télésurveillance la plus utilisée à ce jour au cours de l'épidémie de SARS-CoV-2, bien qu'il existe des solutions similaires dans d'autres régions françaises [26]. Au niveau épidémiologique, Covidom est également une source importante de données sur les patients souffrant d'une forme légère de Covid-19. 
Tableau 1 Caractéristiques générales des adultes enregistrés dans la cohorte Covidom dans le cadre d'une prise en charge ambulatoire $(n=27549)$

\begin{tabular}{|c|c|}
\hline Variable & $n(\%)$ \\
\hline \multicolumn{2}{|l|}{ Caractéristiques générales } \\
\hline Âge, moyenne (ET) & $43,0(13,9)$ \\
\hline \multicolumn{2}{|l|}{ Genre } \\
\hline Homme & $10147(36,8)$ \\
\hline Femme & $17371(63,1)$ \\
\hline \multicolumn{2}{|l|}{ Facteurs de risque } \\
\hline IMC médian (IQR) & $24,9(22,1-28,5)$ \\
\hline Surpoids (ICM : $25-30 \mathrm{~kg} / \mathrm{m}^{2}$ ) & $8237(29,9)$ \\
\hline Obésité (IMC $\left.>30 \mathrm{~kg} / \mathrm{m}^{2}\right)$ & $4987(18,1)$ \\
\hline Tabagisme actif & $4716(17,2)$ \\
\hline \multicolumn{2}{|l|}{ Principales comorbidités } \\
\hline Asthme & $3511(12,8)$ \\
\hline Hypertension & $3457(12,6)$ \\
\hline Diabètes & $1349(4,9)$ \\
\hline Insuffisance cardiaque & $534(1,9)$ \\
\hline $\begin{array}{l}\text { Bronchopneumopathie chronique } \\
\text { obstructive }\end{array}$ & $473(1,7)$ \\
\hline Coronaropathie & $391(1,4)$ \\
\hline Cancer en cours de traitement & $319(1,2)$ \\
\hline Insuffisance rénale chronique & $302(1,1)$ \\
\hline \multicolumn{2}{|l|}{ Symptômes } \\
\hline \multicolumn{2}{|l|}{ Généraux } \\
\hline Asthénie & $23798(86,4)$ \\
\hline Température $\geq 38,5$ degrés & $13559(49,2)$ \\
\hline Frissons & $15311(55,6)$ \\
\hline Myalgies & $15126(54,9)$ \\
\hline \multicolumn{2}{|l|}{ Respiratoires } \\
\hline Toux & $17591(63,9)$ \\
\hline Dyspnée & $13438(48,8)$ \\
\hline Douleur thoracique & $7254(26,5)$ \\
\hline Pression thoracique & $7413(26,9)$ \\
\hline \multicolumn{2}{|l|}{ Gastro-intestinaux } \\
\hline Anorexie & $10960(39,8)$ \\
\hline Nausées/vomissements & $6165(22,4)$ \\
\hline Diarrhées & $9894(35,9)$ \\
\hline \multicolumn{2}{|l|}{ Neurologiques } \\
\hline Anosmie & $8770(31,8)$ \\
\hline Agueusie & $8822(32,0)$ \\
\hline Anosmie ou agueusie & $10301(37,4)$ \\
\hline \multicolumn{2}{|l|}{ Cutanés } \\
\hline Éruptions cutanées & $2628(9,5)$ \\
\hline Engelures & $542(2,0)$ \\
\hline Conjonctivites & $2056(7,5)$ \\
\hline Autres & $3851(14,0)$ \\
\hline
\end{tabular}

ET : écart-type ; IMC : indice de masse corporelle ; IQR : interquartile
Du point de vue des patients, ce système a pu les rassurer grâce au suivi quotidien, mais peu invasif, avec une réponse adaptée en cas d'aggravation. Les informations mises à disposition sur l'application, la disponibilité des intervenants et la rapidité de la réponse en cas d'alerte expliquent aussi en partie le succès de la solution auprès des patients. Ces outils ont également l'avantage d'assurer une surveillance étroite tout en minimisant les contacts physiques, ce qui peut contribuer à limiter la propagation du virus et la contamination éventuelle du personnel de santé. Du point de vue de la santé publique, ce système peut offrir un filet de sécurité, en partie virtuel, afin de détecter rapidement les signes de détérioration chez les patients atteints d'une forme légère de Covid19, tout en préservant les ressources grâce au processus en deux étapes : des algorithmes d'alerte automatisés qui peuvent déclencher, si nécessaire, une réponse médicale. Les algorithmes automatiques et les professionnels de santé se trouvant dans un centre de télésurveillance unique pourraient contribuer à préserver les ressources hospitalières pour les patients qui en ont le plus besoin. Au niveau de l'organisation de l'offre de soins non programmés, le développement et le déploiement de Covidom sont le fruit d'un travail collaboratif entre les professionnels hospitaliers et libéraux (URPS Médecins). À l'aide d'un outil accessible en ligne via un ordinateur, ou sur son téléphone, plus de 60000 personnes ont bénéficié de cette solution de télésurveillance. Il est probable que celle-ci ait participé à limiter l'engorgement des hôpitaux et des services d'urgence. Quand l'état du patient le nécessitait, celui-ci était, après une évaluation médicale en collaboration avec le Samu, directement dirigé vers la structure de soins la plus appropriée. Ce partenariat entre professionnels libéraux et hospitaliers, autour d'une seule pathologie, apparaît comme une importante démonstration de la faisabilité de telles coopérations à plus grande échelle. Ce modèle pourrait guider les travaux des professionnels libéraux et des professionnels de l'urgence hospitalière dans le cadre de la mise en place du service d'accès aux soins (SAS) [27].

L'un des points importants mis en avant par Covidom est que la coconstruction, avec tous les acteurs du soin (ville, hôpital, institutions), a permis une très grande acceptabilité de la solution par les professionnels, mais aussi par les patients. La surveillance d'un tel nombre de patients n'a été possible que grâce à l'implication de plus de 2000 volontaires issus de la société civile, étudiants en santé (médecine, dentaire, maïeutiques, soins infirmiers, kinésithérapie, etc.) ou professionnels de santé non impliqués directement dans la prise en charge des patients Covid-19. La présence de ces bénévoles s'expliquait par l'annulation d'une grande partie des activités courantes du pays du fait du confinement (enseignements, activités médicales et chirurgicales programmées, chômage partiel dans les entreprises et administrations). Cela a rendu possible le travail en commun de 
personnes aux profils très variés, sur l'organisation de la plateforme en temps réel, la surveillance des patients ou encore l'amélioration de la solution Covidom. La présence d'experts en mégadonnées de manière physique ou virtuelle, en support à l'équipe Covidom, a permis un suivi très fin des indicateurs mais aussi une optimisation des algorithmes de surveillance de manière rapide et agile en lien avec les cliniciens. À l'aide de ces bénévoles a aussi été constituée une cellule de rappel, celle-ci ayant la charge d'appeler tous les patients ne répondant pas à leurs questionnaires.

Enfin, Covidom semble être une solution sûre et efficace, avec peu de recours aux hôpitaux et/ou au Samu. Mais, bien entendu, la solution doit être évaluée de manière approfondie, en particulier la capacité des alertes à identifier les patients à haut risque de détérioration et l'impact médicoéconomique de cette solution. Pour ce faire, nous relierons la base de données Covidom au Système national des données de santé (SNDS) ou à l'entrepôt de données de l'APHP afin d'identifier les patients dont l'état s'est détérioré sans être décelé par la solution et la consommation de soins de la population incluse. Il est à noter que l'application Web est gratuite pour les patients et qu'aucune consultation médicale n'a été facturée par la solution Covidom.

Malgré tous ces points encourageants, il existe plusieurs points devant être améliorés. L'application pourrait offrir plus de souplesse aux patients sur la durée de suivi. Celleci s'est révélée un peu courte pour certains patients présentant des symptômes récurrents ou récidivants, alors que d'autres auraient préféré arrêter celui-ci dès la disparition des symptômes. Ensuite, la télésurveillance des patients numériquement fragiles a été possible, mais initialement, la situation de ceux-ci n'était probablement pas suffisamment prise en compte. Il conviendra donc d'activer de manière précoce la solution Covidom Connect, permettant un suivi rapproché des patients ayant des difficultés avec le numérique à l'aide des permanences d'accès aux soins de santé (PASS) hospitalières et des médecins traitants. Puis, la création d'une structure porteuse, administrative (service hospitalier ou unité de gestion) donnerait une plus grande agilité et permettrait de s'adapter avec plus de facilité à la gestion des importants flux de bénévoles. Enfin, l'envoi du dossier Covidom, ou d'une synthèse de celui-ci sous la forme d'un compte rendu, aux médecins traitants pourrait permettre d'intégrer cet outil dans le suivi longitudinal des patients en médecine ambulatoire.

\section{Conclusion}

Covidom est une solution innovante pour la surveillance à domicile des patients atteints d'une forme légère de Covid19. Ce modèle semble efficace et sûr. Il pourrait être transposé dans d'autres contextes et à plus grande échelle.

Remerciements Nous aimerions remercier Alexandre Falzon, Guillaume Fayolle, Fanny Laporte, Amélie Tortel et toute l'équipe Nouveal e-santé pour le développement de l'application et de l'interface du centre régional. Nous aimerions également remercier Laurent Debastard, Alexandre Grenier, Julien Hody, Thomas Penn et l'ensemble de l'URPS Médecins d'Île-de-France pour leur précieuse aide pour le développement et la dissémination de la solution Covidom.

Liens d'intérêts : les auteurs déclarent ne pas avoir de liens d'intérêts.

\section{Éléments remarquables}

- Coconstruction avec tous les acteurs du soin (ville, hôpital, institutions)

- Acceptabilité très forte des professionnels et des patients

- Implication de plus de 2000 volontaires

- Suivi de plus de 60000 patients sur une période de quelques mois

\section{Pistes d'amélioration}

- Durée de suivi adaptative

- Gestion de flux importants de volontaires

- Prévoir des solutions alternatives pour les patients ayant des difficultés avec le numérique

\section{Références}

1. Lu H, Stratton CW, Tang YW (2020) Outbreak of pneumonia of unknown etiology in Wuhan, China: the mystery and the miracle. J Med Virol 92:401-2

2. Worldometer (2020) Coronavirus update (live): 3,006,113 cases and 207,265 deaths from Covid-19 virus pandemic. https:// www.worldometers.info/coronavirus/ (Dernier accès le 21 juillet 2020)

3. Sandford A (2020) Coronavirus: half of humanity on lockdown in 90 countries. https://www.euronews.com/2020/04/02/coronavirus-in-europe-spain-s-death-toll-hits-10-000-after-record-950new-deaths-in-24-hou (Dernier accès le 21 juillet 2020)

4. $\mathrm{Hu} \mathrm{Z}$, Song $\mathrm{C}, \mathrm{Xu} \mathrm{C}$, et al (2020) Clinical characteristics of 24 asymptomatic infections with Covid-19 screened among close contacts in Nanjing, China. Sci China Life Sci 63:706-11

5. Wang X, Fang J, Zhu Y, et al (2020) Clinical characteristics of non-critically ill patients with novel coronavirus infection 
(Covid-19) in a Fangcang Hospital. Clin Microbiol Infect 26:1063-68

6. Zhou F, Yu T, Du R, et al (2020) Clinical course and risk factors for mortality of adult inpatients with Covid-19 in Wuhan, China: a retrospective cohort study. Lancet 395:1054-62

7. Onder G, Rezza G, Brusaferro S (2020) Case-fatality rate and characteristics of patients dying in relation to Covid-19 in Italy. JAMA 323:1775-6

8. Gandhi RT, Lynch JB, Del Rio C (2020) Mild or moderate Covid-19. N Engl J Med (in press)

9. Huang C, Wang Y, Li X, et al (2020) Clinical features of patients infected with 2019 novel coronavirus in Wuhan, China. Lancet 395:497-506

10. Wu C, Chen X, Cai Y, et al (2020) Risk factors associated with acute respiratory distress syndrome and death in patients with coronavirus disease 2019 pneumonia in Wuhan, China. JAMA Intern Med 180:934-43

11. Richardson S, Hirsch JS, Narasimhan M, et al (2020) Presenting characteristics, comorbidities, and outcomes among 5,700 patients hospitalized with Covid-19 in the New York city area. JAMA 323:2052-9

12. Docherty AB, Harrison EM, Green CA, et al (2020) Features of 20,133 UK patients in hospital with Covid-19 using the ISARIC WHO clinical characterisation protocol: prospective observational cohort study. BMJ 369:m1985

13. Chrisafis A (2020) Vu du Royaume-Uni. Le système de santé français au bord de l'effondrement. https://www.courrierinternational.com/article/vu-du-royaume-uni-le-systeme-de-sante-francais-au-bord-de-leffondrement (Dernier accès le 21 juillet 2020)

14. Legido-Quigley H, Mateos-García JT, Campos VR, et al (2020) The resilience of the Spanish health system against the Covid-19 pandemic. Lancet Public Health 5:e251-2

15. Watkins J (2020) Preventing a Covid-19 pandemic. BMJ 368: $\mathrm{m} 810$

16. Armocida B, Formenti B, Ussai S, et al (2020) The Italian health system and the Covid-19 challenge. Lancet Public Health 5:e253
17. Paganini M, Conti A, Weinstein E, et al (2020) Translating Covid-19 pandemic surge theory to practice in the emergency department: how to expand structure. Disaster Med Public Health Prep 27:1-10

18. Wu Z, McGoogan JM (2020) Characteristics of and important lessons from the coronavirus disease 2019 (Covid-19) outbreak in China: summary of a report of 72,314 cases from the Chinese Center for Disease Control and Prevention. JAMA 323:1239-42

19. WHO (2020) Report of the WHO-China joint mission on coronavirus disease 2019 (Covid-19). https:/www.who.int/docs/defaultsource/coronaviruse/who-china-joint-mission-on-covid-19-finalreport.pdf (Dernier accès le 21 juillet 2020)

20. Hollander JE, Carr BG (2020) Virtually perfect? Telemedicine for Covid-19. N Engl J Med 382:1679-81

21. Mahase E (2020) Covid-19: out-of-hours providers are drafted in to manage non-urgent patients in community. BMJ 368:m959

22. Lurie N, Carr BG (2018) The role of telehealth in the medical response to disasters. JAMA Intern Med 178:745-6

23. Keesara S, Jonas A, Schulman K (2020) Covid-19 and health care's digital revolution. N Engl J Med 382:e82

24. Ohannessian R, Duong TA, Odone A (2020) Global telemedicine implementation and integration within health systems to fight the Covid-19 pandemic: a call to action. JMIR Public Health Surveill 6:e18810

25. Santé publique France (2020) Infection au nouveau coronavirus (SARS-CoV-2), Covid-19, France et monde. https://www.santepubliquefrance.fr/dossiers/coronavirus-covid-19 (Dernier accès le 21 juillet 2020)

26. Lifen (2020) Lifen Covid-19 platefrom. https://covid19.lifen.fr/ (Dernier accès le 21 juillet 2020)

27. Ministère des Solidarités et de la Santé (2020) Pacte de refondation des urgences : mise en place du service d'accès aux soins. https://solidarites-sante.gouv.fr/actualites/presse/communiquesde-presse/article/pacte-de-refondation-des-urgences-mise-enplace-du-service-d-acces-aux-soins (Dernier accès le 21 juillet 2020) 